\title{
Factores predictores del consumo de alcohol en adolescentes: datos de un estudio prospectivo de 1 año de seguimiento
}

\section{Predictive factors of alcohol consumption in adolescents: data from 1-year follow-up prospective study}

\author{
Matilde Bousoño*, Susana Al-Halabí**,***, Patricia Burón******, Marlen Garrido*, Eva Mª Díaz-Mesa*, \\ Gonzalo Galván*, Leticia García-Állvarez $* *$, Ángela Velasco*, Camilla Wasserman**************, \\ Vladimir Carli*****, Christina Hoven******, Marco SARChiapone*******, Danuta WaSSERMan*****, \\ Manuel Bousoño***,***, Ma Paz García-Portilla $* * * * * * * * * * * * * * *$, Celso Iglesias $* * * * * * *, * * * * * * * *$, \\ Pilar Alejandra SÁiz*,**************, Julio Bobes*,**************.
}

* Área de Psiquiatría. Universidad de Oviedo; ** Centro de Investigación Biomédica en Red de Salud Mental. CIBERSAM; *** Instituto de Neurociencias del Principado de Asturias. INEUROPA; **** Departamento de Psicología. Universidad de Oviedo; ***** National Centre for Suicide Research and Prevention of Mental Ill-Health, Karolinska Institutet, Stockholm, Sweden; ****** Division of Child and Adolescent Psychiatry and Department of Psychiatry, New York State Psychiatric Institute, Columbia University, New York, NY, USA. Department of Epidemiology, Mailman School of Public Health, Columbia University, New York, NY, USA; ******* Department of Medicine and Health Science, University of Molise, Campobasso, Italy. National Institute for Health, Migration and Poverty, Rome, Italy; ******** Servicio de Salud del Principado de Asturias. SESPA.

\section{Resumen}

El uso/abuso de alcohol es un problema de salud en los adolescentes. La última Encuesta sobre uso de drogas en Enseñanzas Secundarias realizada en España (ESTUDES 2014-2015), pone de manifiesto que $76,8 \%$ de los adolescentes entre 14 y 18 años consumieron alcohol en el último año y $68,2 \%$ en el último mes. El principal objetivo es determinar los factores que se asocian con el consumo de alcohol a medio plazo en una muestra de adolescentes españoles. El estudio forma parte del proyecto Saving and Empowering Young Lives in Europe (SEYLE). La muestra final estuvo compuesta por 708 estudiantes, evaluados en dos momentos temporales [basal $\left(\mathrm{T}_{0}\right)$ y al año $\left(\mathrm{T}_{1}\right)$ ] [varones: $51,98 \%$, edad media basal $(\mathrm{DE})=4,43(0,67)]$. Se realizaron análisis de regresión univariante y multivariante, con el fin de investigar las relaciones entre posibles variables predictoras descritas en el momento temporal $\mathrm{T}_{0}$ y el consumo de alcohol en el momento $\mathrm{T}_{1}$.En el momento basal $\left(\mathrm{T}_{0}\right)$ la prevalencia de abuso de alcohol fue del 25,56\%, mientras que la prevalencia al año fue del 49,72\% ( $\left.\mathrm{T}_{1}\right)$. Las variables que predicen de forma significativa el abuso de alcohol al cabo de un año son: abuso previo del alcohol en el momento $\mathrm{T}_{0}(\mathrm{p}<0,001)$, abuso previo de drogas $(\mathrm{p}=0,011)$, padres que asisten a sus competiciones deportivas $(\mathrm{p}=0,005)$, problemas de relación con compañeros $(p=0,019)$ y ausencia de comportamiento prosocial $(p=0,043)$. A la vista de nuestros resultados se puede concluir que, en adolescentes, los trastornos externalizantes parecen ser factores determinantes de consumo de alcohol a medio plazo.

Palabras clave: Adolescentes; Consumo de Alcohol; Factores predictores; Estudio prospectivo.

\begin{abstract}
Alcohol use/abuse is a health problem in adolescents. The last Survey on use of drugs in Secondary Schoolers carried out in Spain (ESTUDES 2014-2015), reveals that $76.8 \%$ of adolescents aged 14 to 18 years consumed alcohol in the previous year and $68.2 \%$ in the last month. The aim of this study is to determine the medium-term factors associated with alcohol consumption in a sample of Spanish adolescents. The present study was carried out as a part of the Saving and Empowering Young Lives project in Europe (SEYLE) project. The final sample was composed of 708 students, assessed at two times [basal $\left(\mathrm{T}_{0}\right)$ and one year later $\left(\mathrm{T}_{1}\right)$ ] [males: $51.98 \%$, basal mean age $(\mathrm{SD})=4.43(0.67)]$. Univariate and multivariate regression analyses were performed in order to investigate relationships between possible predictive variables found at time $\mathrm{T}_{0}$ and alcohol consumption at time $\mathrm{T}_{1}$. At basal time $\left(\mathrm{T}_{0}\right)$ the prevalence of alcohol abuse was $25.56 \%$, whereas the prevalence one year later was $49.72 \%\left(\mathrm{~T}_{1}\right)$. Variables that significantly predict alcohol abuse within a year are: previous alcohol abuse at $\mathrm{T}_{0}(\mathrm{p}<0.001)$, previous abuse of drugs $(\mathrm{p}=0.011)$, parents attending their sporting events $(p=0.005)$, peer problems $(p=0.019)$, and lack of prosocial behaviour $(\mathrm{p}=0.043)$. In the light of our results, it can be concluded that, in adolescents, externalizing disorders seem to be determining factors of medium-term alcohol consumption. Keywords: Adolescents; Alcohol Consumption; Predictive factors; Follow-up study.
\end{abstract}

Recibido: Julio 2017; Aceptado: Diciembre 2017.

Enviar correspondencia a:

Julio Bobes García. Área de Psiquiatría. Facultad de Medicina. Julián Clavería 6- 3o,33006, Oviedo.

E-mail: bobes@uniovi.es 


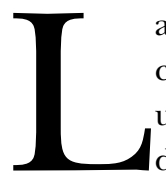
a Organización Mundial de la Salud (OMS) ha calificado el consumo abusivo de alcohol como un problema de salud pública de alcance mundial y ha alertado de los daños que provoca sobre la salud física y psicológica. En 2012, alrededor de 3,3 millones de muertes en todo el mundo fueron causadas por el consumo de alcohol (World Health Organization, 2014). El problema también resulta relevante en el grupo de población joven. Según la última Encuesta Estatal sobre uso de drogas en Enseñanzas Secundarias (ESTUDES 2014-2015), en España un 76,8\% de los adolescentes entre 14 y 18 años consumieron alcohol en el último año y un $68,2 \%$ en el último mes (Plan Nacional sobre Drogas, 2016). A nivel Europeo los datos son similares, con un $79 \%$ de los estudiantes de entre 15 y 16 años que habían consumido alcohol en los últimos 12 meses y un $57 \%$ que lo había hecho en el último mes (Hibell et al., 2012).

Existen datos que demuestran la asociación entre distintas características del grupo de población adolescente y el consumo de alcohol y otras drogas. Por ejemplo, los jóvenes que estudian bachillerato (Patrick, Yeomans-Maldonado y Griffin, 2016) tienen mayor prevalencia de consumo de alcohol, en los años que dura la escolarización, que los no estudiantes, que presentan más consumo de marihuana (Johnston, O’Malley, Miech, Bachman y Schulenberg, 2012; O’Malley y Johnston, 2002). Sin embargo, a largo plazo, las personas que no cursaron bachillerato tienden a tener mayores consumos de alcohol y más problemas de uso de otras sustancias en la etapa adulta, comparados con aquellos que sí estudiaron (Lanza y Collins, 2006; Patrick et al., 2016; White, Labouvie y Papadaratsakis, 2005).

El sexo también es un factor asociado al consumo de alcohol. En edades más jóvenes ( $8^{\circ}$ grado), las chicas presentan tasas de consumo de alcohol en los últimos 30 días (13\%) ligeramente superior a los chicos (12\%). Sin embargo, esta proporción se invierte en los alumnos de más edad $\left(12^{\circ}\right.$ grado), con tasas del 38 y $42 \%$ respectivamente, y se mantiene en la edad adulta, con tasas mayores de consumo en varones que en mujeres (Johnston et al., 2012; Wilsnack et al., 2000).

Además, el consumo de alcohol en adolescentes se ha asociado a problemas conductuales y neuropsicológicos, observándose una asociación entre consumo de alcohol y déficit en la función ejecutiva que se manifiesta en una mayor dificultad para la toma de decisiones, y cuya gravedad correlaciona inversamente con la edad de la primera ingesta etílica (Deckel, Bauer y Hesselbrock, 1995; Kim, Kim y Kwon, 2001; Sobeck, Abbey, Agius, Clinton y Harrison, 2000).

También existen otros factores que se han asociado con el consumo de alcohol en adolescentes. Han presentado correlación negativa con el consumo de alcohol: la supervisión parental (Dever et al., 2012; Pilgrim, Schulenberg, O’Malley, Bachman y Johnston, 2006); la religiosidad (Brown, Schulenberg, Bachman, O’Malley y Johnston,
2001; Wallace et al., 2007; Wray-Lake et al., 2012); el vínculo con la comunidad (Wray-Lake et al., 2012); el nivel de autoestima (Maslowsky y Schulenberg, 2013); y la actitud negativa frente al consumo de alcohol, que resulta ser uno de los factores de prevención más potentes frente al consumo abusivo. Por el contrario, son factores que correlacionan positivamente con el consumo de alcohol: la conducta conflictiva externalizada (Bachman et al., 2008; Maslowsky y Schulenberg, 2013); la búsqueda de sensaciones y toma de riesgo (Dever et al., 2012; Patrick et al., 2010; Pilgrim et al., 2006; Schulenberg et al., 1996); y el estado afectivo depresivo (Maslowsky y Schulenberg, 2013; Patrick et al., 2010; Schulenberg et al., 1996). El ejercicio y el deporte tienen un efecto complejo: mientras realizar ejercicio correlaciona negativamente con el uso de alcohol durante la adolescencia, el participar en deportes de equipo correlaciona positivamente, especialmente en varones (Dever et al., 2012; Terry-McElrath, O’Malley y Johnston, 2011).

De todas las variables estudiadas, la que presenta una asociación más fuerte con el consumo de alcohol y otras drogas es tener amigos que se emborrachan, (Patrick y Schulenberg, 2010); y la que más influye en el pronóstico es la edad de inicio del consumo: los sujetos con abuso de alcohol a edades más tempranas tienen una peor respuesta a los tratamientos y un peor pronóstico (Gueorguieva et al., 2014).

La precocidad en el inicio de consumo de alcohol empeora el pronóstico a largo plazo. En las personas que desarrollarán posteriormente dependencia alcohólica, el consumo intensivo suele comenzar típicamente a una edad temprana (Schulenberg et al., 1996; White, Johnson y Buyske, 2000). El consumo de alcohol tempranamente en la adolescencia, sobre todo cuando se da en individuos de clase socioeconómica baja, aparece asociado a un desarrollo más rápido de la dependencia (Ehlers, Slutske, Gilder, Lau y Wilhelmsen, 2006) y a un mayor riesgo de presentar un abuso de alcohol persistente (Yaogo, Fombonne, Lert y Melchior, 2015). Otro elemento pronóstico negativo es el puesto de manifiesto por Jessor (1991), que muestra que el uso y abuso del alcohol no suele ocurrir de forma aislada, sino que tiende a presentarse asociado a otros problemas del comportamiento adolescente como el uso de drogas ilícitas, el comportamiento antisocial, la conducta sexual precoz y un pobre rendimiento académico.

El objetivo del presente estudio es determinar los factores que se asocian con el consumo de alcohol en una muestra de adolescentes españoles.

\section{Método}

Se llevó a cabo un estudio epidemiológico observacional prospectivo, longitudinal en el que se analizaron los datos españoles del proyecto Saving and Empowering Young Lives in Europe (SEYLE) (Wasserman et al., 2012) en dos momentos de la investigación: evaluación basal $\left(\mathrm{T}_{0}\right)$ y al año $\left(\mathrm{T}_{1}\right)$. 


\section{Participantes}

La muestra (sub-muestra española) en el momento $\left(\mathrm{T}_{0}\right)$ está compuesta por 1026 sujetos, reclutados en 12 centros educativos públicos seleccionados de forma aleatoria entre los existentes en de la Comunidad Autónoma del Principado de Asturias, teniendo en cuenta los criterios de inclusión y exclusión del proyecto SEYLE que figuran a continuación (Bousoño et al., 2017; Wasserman et al., 2010):

Para participar en el estudio, los centros escolares y adolescentes debieron cumplir los siguientes criterios de inclusión: la autoridad escolar se compromete a participar; se trata de centro escolar público no especializadas; el centro cuenta con, al menos, 40 alumnos de 15 años; el centro tiene alumnos de ambos sexos; el centro tiene más de dos maestros para alumnos de 15 años; no más del $60 \%$ de los alumnos en edad de 15 años presentan el mismo sexo; y se obtiene el consentimiento informado de los padres y alumnos.

En el momento basal $\left(\mathrm{T}_{0}\right)$, se estudiaron 1026 adolescentes (edad media, 14,52 años; desviación estándar de 0,702; $51,66 \%$ varones y 48,34\% mujeres). De éstos, 708 estudiantes $(51,84 \%$ varones y $48,16 \%$ mujeres $)$ participaron en el seguimiento realizado un año después del inicio de la investigación ( $\left.\mathrm{T}_{1}\right)$, que corresponde al 69,01\% de la muestra basal. En los momentos $\mathrm{T}_{0} \mathrm{y} \mathrm{T}_{1}$ se utilizaron cuestionarios que los adolescentes cumplimentaron usando lápiz y papel.

\section{SEYLE basal (T0)}

$\mathrm{n}=1026$ edad media: 14,52 años $51,66 \%$ hombres $48,34 \%$ mujeres

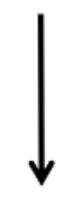

SEYLE seguimiento a un año (T1) $\mathrm{n}=708(69,01 \%$ de la muestra basal $)$ $51,84 \%$ hombres $48,16 \%$ mujeres

Figura 1. Flujograma del número de estudiantes que tomaron parte en cada una de las fases de la investigación (To-T1).

\section{Procedimiento}

En cumplimiento de las normas que rigen la investigación en población joven, previamente al inicio del estudio se obtuvo la autorización del fiscal de menores y la aprobación del Comité Ético de Investigación Clínica del Principado de Asturias. Las autoridades escolares locales otorgaron permiso para acceder a los centros educativos seleccionados y los sujetos de estudio otorgaron el asentimiento y consentimiento informado, según fuera requerido. Se aplicó un cuestionario estructurado de autoinforme a los sujetos participantes en dos momentos temporales distintos $\left(\mathrm{T}_{0}=\right.$ basal y $\mathrm{T}_{1}=1$ año $)$, se asignó un código único a los adolescentes reclutados con el fin de llevar a cabo el seguimiento a nivel individual, y no sólo de grupo. La codificación de los datos protege la privacidad de los sujetos del estudio y la base de datos fue anónima, siendo imposible la identificación de las personas. La correspondencia entre los códigos anónimos y los sujetos de estudio se guardó de manera confidencial en un lugar independiente, adecuadamente custodiado y habilitado para ello en el Área de Psiquiatría de la Universidad de Oviedo, siendo el investigador principal del proyecto el responsable de esos datos. Todos los aspectos concernientes a control de calidad, homogeneización de procedimiento, aspectos éticos y validez de las escalas utilizadas se describen detalladamente en el trabajo de Carli et al. (2013).

\section{Evaluación del estudio}

Las evaluaciones fueron realizadas en horario escolar y recogen datos como la edad, el país de nacimiento del adolescente y sus padres, la situación laboral de los padres, estilos de vida, familia, convivencia, salud mental y suicidalidad.

Se investigó el abuso del alcohol a través del ítem (“ $A$ lo largo de tu vida, ¿cuántas veces has bebido tanto alcohol que estuviste realmente borracho?") en los dos momentos temporales considerados $\mathrm{T}_{0}$ y $\mathrm{T}_{1}$ enfrentando la respuesta nunca frente al resto de las categorías (1 ó 2 veces, 3 a 9 veces, 10 o más veces).

La ideación y conducta suicida fueron medidas por medio de la escala de suicidio de Paykel (PSS) (Paykel, Myers, Lindenthal y Tanner, 1974). El PSS comprende las siguientes cinco preguntas: durante las últimas 2 semanas: (i) ¿Has sentido que la vida no merece la pena?, (ii) ¿Has deseado estar muerto?, (iii) ¿Has pensado en quitarte la vida aunque realmente no lo fueras a hacer?, (iv) ¿Has llegado al punto en el que consideraras realmente quitarte la vida o hicieses planes sobre cómo lo harías?; y (v) ¿Alguna vez has intentado quitarte la vida?. Se consideró que la persona tenía pensamientos suicidas si contestaba "si" a la tercera (iii) o la cuarta (iv) cuestión del PSS. Los intentos de suicidio fueron definidos por la respuesta "si" a la última pregunta (v) del PSS.

Para la valoración del consumo de sustancias se ha utilizado el cuestionario Global School-based Student Health Survey-GSHS- (World Health Organization, 2015), estableciendo los siguientes puntos de corte: en el consumo de alcohol se ha considerado como punto de corte el consumir cualquier cantidad de alcohol dos veces o más a la semana; en el consumo de drogas, haber usado drogas ilegales al menos tres veces a lo largo de la vida y en el consumo de tabaco fumar más de diez cigarrillos al día. Este mismo cuestionario ha sido utilizado para evaluar variables como comportamientos alimentarios, factores protectores, actividad física, salud mental, etc. Los ítems del cuestionario GSHS fueron recodificados para identificar estas áreas de 
comportamiento considerando sueño reducido dormir 6 horas por la noche o menos, sobrepeso (índice de masa corporal IMC por encima del percentil 95 para la edad), bajo peso (IMC por debajo del percentil 5 para la edad), comportamiento sedentario: realizar actividad física menos de una vez por semana, uso de medios (usar internet, TV y videojuegos por razones no relacionadas con la escuela o el trabajo durante 5 horas o más por día), absentismo escolar: saltarse la escuela al menos una vez por semana sin estar enfermo o teniendo otra excusa legítima (Carli et al., 2014).

Se utilizó el inventario de Beck (BDI-II) para evaluar los síntomas depresivos (Beck, Steer, Ball y Ranieri, 1996), considerando que una puntuación igual o mayor de 20 suponía riesgo de depresión. El Inventario de depresión de Beck (BDI-II) es un cuestionario de 21 ítems usado para medir síntomas depresivos. Para el presente estudio se ha usado una versión modificada del BDI-II donde el ítem "pérdida de la libido" fue retirado de la escala ya que se consideró una pregunta inapropiada para la población adolescente. La evidencia muestra que la omisión de esta pregunta no afecta a la fiabilidad o validez al instrumento (Byrne, Stewart y Lee, 2004).

Para evaluar la psicopatología se empleó el Cuestionario de Capacidades y Dificultades (SDQ) (Goodman, Meltzer y Bailey, 2003) que valora síntomas emocionales, problemas de conducta, hiperactividad/falta de atención, problemas de relación entre pares y comportamiento pro-social. Los puntos de corte seleccionados fueron: una puntuación mayor o igual que 7 para los síntomas emocionales, una puntuación mayor o igual que 5 para los problemas de conducta y una puntuación mayor o igual que 7 para la hiperactividad. En el caso de los problemas con los compañeros, el punto de corte se fijó en una puntuación mayor o igual que 6 , mientras que la falta de comportamiento pro-social se definió con una puntuación menor o igual que 4 (Carli et al., 2013).

Para valorar la ansiedad se utilizó el Cuestionario de Ansiedad de Zung (Zung, 1971), el cual consta de 20 ítems, en función de los puntos en las respuestas se les asignará de 1 a 4 y se sumarán, la puntuación total oscila entre 20 y 80 puntos siendo el punto de corte 30 puntos o más.

\section{Análisis estadístico}

Se empleó estadística descriptiva para determinar la prevalencia del consumo de alcohol en los diferentes puntos temporales, así como las frecuencias de las distintas variables psicosociales (ejemplo: empleo de los padres, padres comprensivos, comportamiento suicida, etc). También se calcularon los valores medios y desviaciones estándar de las variables de escala (ejemplo: dimensiones de las escala SDQ, puntuación en las escala de depresión BDI, escala de ansiedad de Zung, etc.).

Se realizaron análisis de regresión univariante y multivariantes, con el fin de investigar las relaciones entre los predictores anteriormente descritos en el momento temporal $\mathrm{T}_{0}$ y el consumo de alcohol en el momento $\mathrm{T}_{1}$. Para el análisis de regresión múltiple, se usó la metodología stepwise minimizando el valor del criterio de información bayesiano (BIC). Todos los análisis estadísticos se realizaron con la versión 24 del software SPSS. Se eligió un nivel de significación alfa del $5 \%$.

\section{Resultados}

Los siguientes resultados se refieren a una cohorte de 708 estudiantes que fueron evaluados en los dos momentos de la investigación. En el momento basal $\left(\mathrm{T}_{0}\right)$, la edad media era de 14,43 años con una desviación estándar (DE) de 0,67 y un rango de edad entre los 13 y los 17 años, 367 estudiantes eran varones $(51,98 \%)$ y 339 mujeres $(48,02 \%)$. En el momento basal $\left(\mathrm{T}_{0}\right)$ la prevalencia de abuso de alcohol fue del 25,56\%, mientras que la prevalencia al año fue del $49,72 \%\left(\mathrm{~T}_{1}\right)$. La Tabla 1 muestra la proporción de estudiantes que presentaron abuso del alcohol en los puntos temporales considerados en el estudio.

Los resultados de las regresiones separadas de cada una de las características estudiadas en el momento temporal $\mathrm{T}_{0}$ frente al abuso de alcohol en el momento $\mathrm{T}_{1}$ se presentan en la Tabla 2. Dicho análisis de regresión, efectuado variable a variable para todas las variables independientes consideradas, mostró resultados significativos para las variables: abuso de alcohol y abuso de las drogas en $\mathrm{T}_{0}$, cuestionario SDQ (problemas de conducta), escala de depresión BDI y comportamiento suicida. Los resultados del análisis de regresión múltiple se presentan en la Tabla 3. Después de aplicar el procedimiento de regresión por pasos, las siguientes variables resultaron predictores significativos del abuso de alcohol en $\mathrm{T}_{1}$ : abuso del alcohol en $\mathrm{T}_{0}(\mathrm{~b}=0,34$, $\mathrm{p}<0,001)$, abuso de drogas en $\mathrm{T}_{0}(\mathrm{~b}=0,106, \mathrm{p}=0,011)$, y padres que han visto una actuación o competición deportiva en $\mathrm{T}_{0}(\mathrm{~b}=0,113, \mathrm{p}=0,005)$, SDQ problemas de relación con los colegas en $\mathrm{T}_{0}(\mathrm{~b}=0,092, \mathrm{p}=0,019)$, comportamiento prosocial en $\mathrm{T}_{0}(\mathrm{~b}=-0,079, \mathrm{p}=0,043)$. El $\mathrm{R}^{2}$ ajustado del modelo final fue de 0,287 con un valor $F$ para dicho modelo de 2129,78 lo que nos permite rechazar la hipótesis nula de que en dicho modelo de regresión todos los coeficientes de las variables independientes sean iguales a cero. Las otras variables consideradas no se incluyeron en el modelo final de predicción del abuso de alcohol en $\mathrm{T}_{1}$. 
Tabla 1. Abuso de alcohol (basal y un año)

\begin{tabular}{cccc}
\hline Basal & $\mathbf{1}$ año & N & (\%) \\
\hline no & no & 356 & $50,28 \%$ \\
no & sí & 171 & $24,16 \%$ \\
sí & sí & 181 & $25,56 \%$ \\
\hline
\end{tabular}

Tabla 2. Modelos de regresiones separadas de abuso del alcohol después de un año para cada uno de los factores demográficos, sociales y psicológicos considerados en el momento $T_{0}$.

\begin{tabular}{|c|c|c|c|}
\hline \multirow{3}{*}{ Factores demográficos, sociales y psicológicos } & \multicolumn{3}{|c|}{ Abuso del alcohol en $\mathrm{T}_{1}$} \\
\hline & \multicolumn{2}{|l|}{$\mathbf{T}_{\mathrm{o}}$} & \multirow[t]{2}{*}{$\mathbf{R}^{2}$} \\
\hline & $\beta$ (IC 95\%) & $\mathbf{P}$ & \\
\hline Abuso del alcohol & $0,186(0,163 ; 0,203)$ & $<0,001$ & 0,345 \\
\hline \multicolumn{4}{|l|}{ Composición del hogar } \\
\hline Los estudiantes viven con un padre o pariente biológico & $-0,060(-0,160 ; 0,400)$ & 0,109 & 0,04 \\
\hline \multicolumn{4}{|l|}{ Implicación parental } \\
\hline Padres comprobaron que hacía los deberes & $-0,087(-0,177 ; 0,003)$ & 0,066 & 0,029 \\
\hline Padres comprensivos con problemas o preocupaciones & $0,014(-0,006 ; 0,034)$ & 0,766 & 0,054 \\
\hline Padres saben lo que realmente hace con su tiempo libre & $-0,066(-0,616 ; 0,484)$ & 0,172 & 0,073 \\
\hline Padres ayudan a tomar decisiones & $-0,059(-0,509 ; 0,391)$ & 0,201 & 0,019 \\
\hline Padres buscan tiempo para hablar sobre las cosas que le pasan & $-0,051(-0,147 ; 0,045)$ & 0,263 & 0,028 \\
\hline $\begin{array}{l}\text { Padres van a verte cuando haces alguna actividad especial como jugar un partido o realizar } \\
\text { alguna actuación }\end{array}$ & $0,005(-0,325 ; 0,335)$ & 0,917 & 0,01 \\
\hline Padres prestan atención a tus opiniones o a lo que dices & $-0,043(-0,721 ; 0,635)$ & 0,359 & 0,02 \\
\hline \multicolumn{4}{|l|}{ Desempleo parental } \\
\hline Desempleo de los padres o tutores del alumno & $0,059(-0,513 ; 0,631)$ & 0,141 & 0,003 \\
\hline \multicolumn{4}{|l|}{ Cuestionario de capacidades y dificultades(SDQ) } \\
\hline Síntomas emocionales (SDQ) & $0,088(-0,003 ; 0,179)$ & 0,098 & 0,017 \\
\hline Problemas de conducta (SDQ) & $0,141(0,131 ; 0,151)$ & 0,002 & 0,077 \\
\hline Hiperactividad / falta de atención (SDQ) & $-0,027(-0,116 ; 0,062)$ & 0,557 & 0,014 \\
\hline Problemas con los compañeros (SDQ) & $0,041(-0,205 ; 0,287)$ & 0,357 & 0,001 \\
\hline Comportamiento prosocial (SDQ) & $-0,051(-0,113 ; 0,011)$ & 0,313 & 0,024 \\
\hline Depresión (escala BDI) & $0,193(0,161 ; 0,225)$ & $<0,001$ & 0,097 \\
\hline Ansiedad (escala de Zung) & $-0,001(0,693 ; 0,691)$ & 0,972 & 0,071 \\
\hline Comportamiento suicida & $0,12(0,114 ; 0,126)$ & 0,04 & 0,057 \\
\hline
\end{tabular}


M. Bousoño, S. Al-Halabí, P. Burón, M. Garrido, E. M. Díaz-Mesa, G. Galván, L. García-Álvarez, Á. Velasco, C. Wasserman, V. Carli, C. Hoven, M. Sarchiapone, D. Wasserman, M. Bousoño, M. P. García-Portilla, C. Iglesias, P. A. Sáiz, J. Bobes.

Tabla 3. Modelo de regresión múltiple de la variable abuso del alcohol después de un año para cada uno de los factores demográficos, sociales y psicológicos considerados en el momento $T_{\text {. }}$.

\begin{tabular}{|c|c|c|}
\hline \multirow{3}{*}{ Factores demográficos, sociales y psicológicos } & \multicolumn{2}{|c|}{ Abuso del alcohol en $\mathrm{T}_{1}$} \\
\hline & \multicolumn{2}{|c|}{$T_{0}$} \\
\hline & $\beta$ (IC 95\%) & p \\
\hline Abuso del alcohol & $0,340(0,16 ; 0,52)$ & $<0,001$ \\
\hline Abuso de las drogas & $0,106(0,021 ; 0,191)$ & 0,011 \\
\hline \multicolumn{3}{|l|}{ Composición del hogar } \\
\hline Los estudiantes viven con un padre o pariente biológico & $-0,030(-0,260 ; 0,200)$ & 0,446 \\
\hline \multicolumn{3}{|l|}{ Implicación parental } \\
\hline Padres comprobaron que hacía los deberes & $-0,018(-0,208 ; 0,172)$ & 0,663 \\
\hline Padres comprensivos con problemas o preocupaciones & $0,051(-0,009 ; 0,111)$ & 0,25 \\
\hline Padres saben lo que realmente hace con su tiempo libre & $0,039(-0,511 ; 0,589)$ & 0,444 \\
\hline Padres ayudan a tomar decisiones & $0,043(-0,327 ; 0,413)$ & 0,323 \\
\hline Padres buscan tiempo para hablar sobre las cosas que le pasan & $-0,002(-0,085 ; 0,089)$ & 0,954 \\
\hline $\begin{array}{l}\text { Padres van a verte cuando haces alguna actividad especial como jugar un partido o realizar } \\
\text { alguna actuación }\end{array}$ & $0,113(0,015 ; 0,211)$ & 0,005 \\
\hline Padres prestan atención a tus opiniones o a lo que dices & $0,048(-0,630 ; 0,726)$ & 0,265 \\
\hline \multicolumn{3}{|l|}{ Desempleo parental } \\
\hline Desempleo de los padres o tutores del alumno & $0(-0,472 ; 0,472)$ & 0,995 \\
\hline \multicolumn{3}{|l|}{ Cuestionario de Capacidades y Dificultades (SDQ) } \\
\hline Síntomas emocionales (SDQ) & $0,046(-0,864 ; 0,956)$ & 0,372 \\
\hline Problemas de conducta (SDQ) & $0,059(-0,141 ; 0,259)$ & 0,155 \\
\hline Hiperactividad / falta de atención (SDQ) & $-0,007(-0,587 ; 0,573)$ & 0,857 \\
\hline Problemas con los compañeros (SDQ) & $0,092(0,064 ; 0,120)$ & 0,019 \\
\hline Comportamiento prosocial (SDQ) & $-0,079(-0,135 ;-0,023)$ & 0,043 \\
\hline Depresión (escala BDI) & $0,088(-0,001 ; 0,177)$ & 0,058 \\
\hline Ansiedad (escala de Zung) & $0,042(-0,009 ; 0,093)$ & 0,315 \\
\hline Comportamiento suicida & $0,029(-0,008 ; 0,066)$ & 0,478 \\
\hline
\end{tabular}




\section{Discusión}

Estos resultados, en general, son similares a los encontrados por otros autores en población adolescente, aunque con algunas diferencias relevantes. La tasa de abuso de alcohol obtenida en la evaluación basal del presente estudio $(25,56 \%)$ es superior a las tasas de consumo de alcohol en adolescentes europeos que varía entre el 6 y 23\% (Green, Leyland, Sweeting y Benzeval, 2013; Richter, Kuntsche, de Looze y Pförtner, 2013), y triplica las tasas obtenidas por dos trabajos anteriores realizados con metodología similar al actual. En el primero, realizado sobre una muestra con una edad media de 14,9 años (Rüütel et al., 2014), el abuso de alcohol (consumir alcohol 2 o más veces a la semana) encontrado fue del 8,2 \% y en el segundo (Carli et al., 2014), las tasas de abuso de alcohol en los adolescentes de 14 y 15 años, fueron respectivamente de 5,2 y 7,3 $\%$. En nuestro caso, el incremento significativo de la tasa de consumo observado un años después de la observación basal $(49,72 \%)$ podría significar que se ha identificado un periodo muy relevante para el inicio en el consumo de alcohol de la población estudiada.

Se sabe que los patrones individuales de consumo de bebidas alcohólicas están influenciados por los cambios neurológicos, cognitivos y sociales que suceden típicamente en la adolescencia y también que el consumo de alcohol influye en la aparición daño neurológico y deterioro social en la adolescencia (Brown et al., 2008). A pesar de ello, la elevada frecuencia de consumo de alcohol en la población joven hace que el fenómeno se haya banalizado a pesar de la reiteración de datos españoles (ESTUDES 2014-2015), europeos, o estadounidenses, en los que se observa que a los 16 años casi dos tercios de los jóvenes han probado el alcohol al menos una vez en su vida, y más de dos quintos han referido haberse emborrachado al menos una vez; y a los 18 años más de tres cuartas partes de los jóvenes han probado el alcohol al menos una vez en su vida y más de tres quintos han manifestado haberse emborrachado al menos una vez (Johnston, Bachman y Schulenberg, 2012). Nuestros datos confirman el fenómeno añadiendo otro elemento negativo: el acceso al consumo de alcohol se hace a edad muy temprana (en el momento $\mathrm{T}_{0}$, con una edad media 14,4 años, en torno a una cuarta parte de los jóvenes ya habían abusado del alcohol y, entre los que no habían consumido al principio, un porcentaje relevante accedió al alcohol durante el año que duró el periodo de observación del estudio).

Debe señalarse la amplia variabilidad de la tasa de consumo de alcohol en adolescentes entre diferentes países, que podría deberse a factores socioeconómicos, educativos, legales y culturales (Kuntsche et al., 2014). En el caso de presente estudio debe reseñarse, que la población española estudiada pertenece a una sola comunidad autónoma (Asturias), cuyas características sociodemográficas (decrecimiento y envejecimiento poblacional, elevada tasa de paro y empobrecimiento económico), podrían explicar un mayor recurso al consumo de alcohol, como método de evasión en adolescentes de unas circunstancias sociales y unas perspectivas futuras negativas.

En cualquier caso, la observación de una tasa de consumo de alcohol elevada a una edad tan temprana resulta muy importante y no puede ser obviada, ya que la precocidad en el inicio del consumo se asocia con mayor riesgo de desarrollar un Trastorno por Consumo de Alcohol (DeWit, Adlaf, Offord y Ogborne, 2000; Pitkänen, Lyyra y Pulkkinen, 2005).

La situación familiar se ha mostrado como un elemento clave en el contacto de los menores con el alcohol. Un estudio realizado en España muestra como posible "antecedente" un mayor consumo en el entorno familiar, especialmente por parte de los hermanos/as (Golpe, Isorna, Barreiro, Braña y Rial, 2017). Otra investigación reciente revela cómo los patrones de consumo de alcohol de los adolescentes están relacionados con su estructura familiar, siendo un factor protector que el adolescente viva en una familia con padres biológicos frente a una familia monoparental o en una familia con un padre biológico y un padrastro (Rüütel et al., 2014).

Nuestro trabajo encuentra datos que parecen contraintuitivos. Se observa correlación entre la presencia de los padres en las actividades deportivas de los hijos y el consumo de alcohol de los adolescentes al año de seguimiento. En principio, que los padres acompañen a los hijos a las actividades deportivas es una forma de monitorización parental, y la monitorización parental es un factor protector sobre el abuso de alcohol y drogas (Fletcher, Steinberg y Williams-Wheeler, 2004; Tilton-Weaver, Burk, Kerr y Stattin, 2013), al retrasar el comienzo del uso y reducir el riesgo de mal uso (DeVore y Ginsburg, 2005), al mismo tiempo que por lo que el consumo de alcohol tiende a incrementarse con la separación de los padres y la disminución de la monitorización parental (Barnes, Reifman, Farrell y Dintcheff, 2000).

Esta paradoja podría estar justificada, en parte, por el sesgo derivado de la edad, ya que el consumo de alcohol aumenta con la edad (Johnston, O'Malley, Miech, Bachman y Schulenberg, 2016). No obstante, la asociación entre eventos deportivos y consumo de alcohol podría deberse a: las celebraciones con alcohol tras las victorias deportivas son frecuentes; o a la participación en competiciones conlleva a veces el viajar fuera del domicilio paterno, lo cual podría incrementar la probabilidad de consumos alcohólicos (Tahiraj et al., 2016). En cualquier caso, la asociación entre consumo de alcohol y deporte en jóvenes no está clara, con algunos estudios que encuentran que el consumo peligroso de alcohol es más frecuente en chicos que han participado mayor tiempo en deportes $(\mathrm{OR}=1,49)$ (Sajber, Tahiraj, Zenic, Peric y Sekulic, 2016) y otros que muestran un efecto positivo del deporte en jóvenes que se concreta 
en menos consumo de alcohol (Lopez, Rodriguez, Garcia y Perez, 2016).

En jóvenes, los comportamientos perturbadores se asocian con trastornos por uso de alcohol o drogas. Se ha descrito un modelo de desarrollo de trastornos en el cual los problemas parentales y las experiencias de abuso o de abandono en la infancia se combinan con problemas en la escuela y con los compañeros en la adolescencia aumentando el riesgo de abuso de substancias en la etapa adulta precoz (Abrantes, Brown y Tomlinson, 2003; Bifulco, Schimmenti, Jacobs, Bunn y Rusu, 2014). Un estudio reciente realizado en España encuentra asociación estadísticamente significativa entre victimización y consumo de sustancias psicoactivas (Caravaca, Navarro-Zaragoza, Luna, Falcón y Luna, 2017).

Hay estudios que muestran que los niños y adolescentes que experimentan un rechazo por su grupo de compañeros, debido a sus alteraciones de comportamiento, tienden a relacionarse entre ellos (Coie, Terry, Lenox, Lochman y Hyman, 1995; Laird, Jordan, Dodge, Pettit y Bates, 2001) y esta asociación con grupos de compañeros con alteraciones de conducta, media la asociación entre los síntomas depresivos experimentados a los 14 años y un uso de alcohol de riesgo a los 16 años (Pesola et al., 2015). También se ha observado que el uso precoz de alcohol está asociado con el comportamiento antisocial (Brown, 1993; Hill, White, Chung, Hawkins y Catalano, 2000). Se considera que el uso de alcohol en la adolescencia incrementa la probabilidad de otras conductas problemáticas debido a que la exposición al alcohol altera el curso del desarrollo adolescente (Kandel et al., 1999). Para otros autores, sin embargo, (Vanyukov et al., 2003), la comorbilidad es el producto de una predisposición general a contravenir las normas sociales; según esta teoría, el uso de alcohol y la aparición de otras conductas problemáticas serían un resultante de la estructura caracterial. En un estudio reciente se aportan evidencias empíricas entre el consumo de alcohol y otras drogas y el uso problemático de Internet en adolescentes (Golpe, Gómez, Braña, Varela y Rial, 2017).

En estudios realizados en muestras comunitarias de adolescentes, la presencia de uso de alcohol o substancias multiplica por tres las tasas de trastornos comórbidos de salud mental respecto a los jóvenes no consumidores (Kandel et al., 1999). Se sabe que el consumo de alcohol tiende a aumentar durante la adolescencia tardía (McCambridge, McAlaney y Rowe, 2011) y que los problemas relacionados con el alcohol presentes en esta etapa se correlacionan de forma robusta con una mayor morbimortalidad (Hingson, Zha y Weitzman, 2009).

Depresión y consumo de alcohol parecen tener una relación bidireccional en la adolescencial. El uso precoz de alcohol se ha asociado con la depresión (Brook, Brook, Zhang, Cohen y Whiteman, 2002; Wells, Horwood y Fer- gusson, 2004) y la depresión experimentada en la adolescencia ha sido identificada como un factor de riesgo para un mayor consumo de alcohol en esta edad (Marmorstein, Iacono y Malone, 2010; Saraceno, Heron, Munafo, Craddock y Van den Bree, 2012). Un estudio reciente de Pesola et al. (2015) encuentra que la presencia de síntomas depresivos a los 14 años estaría asociada positivamente con una ingesta alcohólica dañina a los 19 años.

Las chicas adolescentes tienen mayor riesgo que los chicos de ser víctimas de abusos (Champion et al., 2004) y de padecer depresión o ansiedad (Poulin, Hand, Boudreau y Santor, 2005) lo que en las chicas podrían dar como resultado el consumo alcohólico mientras que en chicos es más probable que produjera comportamientos externalizantes como los trastornos de conducta e impulsividad (Caspi, Moffitt, Newman y Silva, 1996). Intervenciones psicológicas estructuradas para tratar los problemas de conducta precoces tempranos se han mostrado eficaces; los pacientes tratados muestran actitudes menos favorables hacia las drogas, menor intención de consumo, menor frecuencia de consumo de tabaco y menor intensidad de consumo de alcohol (Romero, Rodríguez, Villar y Gómez-Fraguela, 2016).

El presente estudio presenta algunas limitaciones, forma parte de un estudio de alcance europeo y el tamaño de la muestra no permite establecer relaciones entre variables poco frecuentes, como el consumo de drogas o realizar un estudio segregado por grupos de edad.

\section{Conclusiones}

Nuestro estudio ratifica el elevado consumo de alcohol en población adolescente. Además, pone de manifiesto que variables como el abuso previo de alcohol y/o drogas, los problemas de relación con los colegas y la ausencia de comportamiento prosocial son predictores del abuso de alcohol a medio plazo. Por otra parte, parecen sólidas las relaciones entre consumo de alcohol y trastornos afectivos, aunque nuestros resultados no nos permiten inferir causalidad. Se considera que todos estos factores han de tenerse en cuenta a la hora de diseñar y poner en marcha estrategias de tipo preventivo.

\section{Reconocimientos}

El Proyecto SEYLE está apoyado por la Unión Europea a través del Séptimo Programa Marco (FP7), mediante acuerdo de subvención número HEALTH-F2.2009-223091. Los autores desean agradecer a todos los investigadores y personal que participa en el Programa SEYLE.

\section{Conflicto de intereses}

Los autores declaran que no existe conflicto de interés. 


\section{Referencias}

Abrantes, A. M., Brown, S. A. y Tomlinson, K. L. (2003). Psychiatric comorbidity among inpatient substance abusing adolescents. Journal of Child E Adolescent Substance Abuse, 13, 83-101. doi:10.1300/J029v13n02_04.

Bachman, J., O’Malley, P. M., Schulenberg, J. E., Johnston, L., Freedman-Doan, P. y Messersmith. E. E. (2008). The Education-Drug Use Connection: How Successes and Failures in School Relate to Adolescent Smoking, Drinking, Drug Use, and Delinquency. New York: Lawrence Erlbaum Associates. Recuperado de http://www.psc.isr.umich.edu/ pubs/abs/4465.

Barnes, G. M., Reifman, A. S., Farrell, M. P. y Dintcheff, B. A. (2000). The effects of parenting on the development of adolescent alcohol misuse: A six-wave latent Growth model. Journal of Marriage and Family, 62, 175186. doi:10.1111/j.1741-3737.2000.00175.x.

Beck, A. T., Steer, R. A., Ball, R. y Ranieri, W. (1996). Comparison of Beck Depression Inventories -IA and -II in psychiatric outpatients. Journal of Personality Assessment, 67, 588-597. doi:10.1207/s15327752jpa6703_13.

Bifulco, A., Schimmenti, A., Jacobs, C., Bunn, A. y Rusu, A. C. (2014). Risk factors and psychological outcomes of bullying victimization: A community-based study. Child Indicators Research, 7, 633-648. doi:10.1007/s12187-0149236-8.

Bousoño, M., Al-Halabí, S., Burón, P., Garrido, M., Díaz-Mesa, E. M., Galván, G.,... Bobes, J. (2017). Uso y abuso de sustancias psicotrópicas e internet, psicopatología e ideación suicida en adolescentes. Adicciones, 29, 97-104. doi:10.20882/adicciones.811.

Brook, D. W., Brook, J. S., Zhang, C., Cohen, P. y Whiteman, M. (2002). Drug use and the risk of major depressive disorder, alcohol dependence, and substance use disorders. Archives of General Psychiatry, 59, 1039-1044.

Brown, S. A., McGue, M., Maggs, J., Schulenberg, J., Hingson, R., Swartzwelder, S.,... Murphy, S. (2008). A developmental perspective on alcohol and youths 16 to 20 years of age. Pediatrics, 121, S290-310. doi:10.1542/ peds.2007-2243D.

Brown, S. A. (1993). Recovery patterns in adolescent substance abuse. En Addictive behaviors across the life span: Prevention, treatment, and policy issues, (Baer, John Samuel (Ed); Marlatt, G. Alan (Ed); McMahon, Robert Joseph (Ed). (1993)., p. (161-183).). Thousand Oaks, CA. Recuperado de http://psycnet.apa.org/psycinfo/1993-97504-007.

Brown, T. N., Schulenberg, J., Bachman, J. G., O’Malley, P. M. y Johnston, L. D. (2001). Are risk and protective factors for substance use consistent across historical time?: National data from the high school classes of 1976 through 1997. Prevention Science, 2, 29-43.

Byrne, B. M., Stewart, S. M. y Lee, P. W. H. (2004). Validating the beck depression inventory-II for Hong Kong
Community Adolescents. International Journal of Testing, 4, 199-216. doi:10.1207/s15327574ijt0403_1.

Caravaca, F., Navarro-Zaragoza, J., Luna, A., Falcón, M. y Luna, A. (2017). Asociación entre victimización por bullying y consumo de sustancias entre la población universitaria de España. Adicciones, 29, 22-32. doi:10.20882/ adicciones.827.

Carli, V., Wasserman, C., Wasserman, D., Sarchiapone, M., Apter, A., Balazs, J.,... Hoven, C. W. (2013). The saving and empowering young lives in Europe (SEYLE) randomized controlled trial (RCT): methodological issues and participant characteristics. BMC Public Health, 13, 479. doi:10.1186/1471-2458-13-479.

Carli, V., Hoven, C. W., Wasserman, C., Chiesa, F., Guffanti, G., Sarchiapone, M.,... Wasserman, D. (2014). A newly identified group of adolescents at "invisible" risk for psychopathology and suicidal behavior: findings from the SEYLE study. World Psychiatry, 13, 78-86. doi: 10.1002/ wps. 20088.

Caspi, A., Moffitt, T. E., Newman, D. L. y Silva, P. A. (1996). Behavioral observations at age 3 years predict adult psychiatric disorders. Longitudinal evidence from a birth cohort. Archives of General Psychiatry, 53, 1033-1039. doi:10.1001/archpsyc.1996.01830110071009.

Champion, H. L. O., Foley, K. L., DuRant, R. H., Hensberry, R., Altman, D. y Wolfson, M. (2004). Adolescent sexual victimization, use of alcohol and other substances, and other health risk behaviors. The Journal of Adolescent Health, 35, 321-328. doi:10.1016/j.jadohealth.2003.09.023.

Coie, J., Terry, R., Lenox, K., Lochman, J. y Hyman, C. (1995). Childhood peer rejection and aggression as predictors of stable patterns of adolescent disorder. $D e^{-}$ velopment and Psychopathology, 7, 697-713. doi:10.1017/ S0954579400006799.

Deckel, A. W., Bauer, L. y Hesselbrock, V. (1995). Anterior brain dysfunctioning as a risk factor in alcoholic behaviors. Addiction, 90, 1323-1334. doi:10.1046/j.13600443.1995.901013234.x.

Dever, B. V., Schulenberg, J. E., Dworkin, J. B., O’Malley, P. M., Kloska, D. D. y Bachman, J. G. (2012). Predicting risk-taking with and without substance use: the effects of parental monitoring, school bonding, and sports participation. Prevention Science, 13, 605-615. doi:10.1007/ s11121-012-0288-z.

DeVore, E. R. y Ginsburg, K. R. (2005). The protective effects of good parenting on adolescents. Current Opinion in Pediatrics, 17, 460-465.

DeWit, D. J., Adlaf, E. M., Offord, D. R. y Ogborne, A. C. (2000). Age at first alcohol use: a risk factor for the development of alcohol disorders. American Journal of Psychiatry, 157, 745-750. doi:10.1176/appi.ajp.157.5.745.

Ehlers, C. L., Slutske, W. S., Gilder, D. A., Lau, P. y Wilhelmsen, K. C. (2006). Age at first intoxication and alcohol 
M. Bousoño, S. Al-Halabí, P. Burón, M. Garrido, E. M. Díaz-Mesa, G. Galván, L. García-Álvarez, Á. Velasco, C. Wasserman, V. Carli, C. Hoven, M. Sarchiapone, D. Wasserman, M. Bousoño, M. P. García-Portilla, C. Iglesias, P. A. Sáiz, J. Bobes.

use disorders in Southwest California Indians. Alcoholism, Clinical and Experimental Research, 30, 1856-1865. doi:10.1111/j.1530-0277.2006.00222.x.

Fletcher, A. C., Steinberg, L. y Williams-Wheeler, M. (2004). Parental influences on adolescent problem behavior: revisiting Stattin and Kerr. Child Development, 75, 781-796. doi:10.1111/j.1467-8624.2004.00706.x.

Golpe, S., Gómez, P., Braña, T., Varela, J. y Rial, A. (2017). Relación entre el consumo de alcohol y otras drogas y el uso problemático de Internet en adolescentes. Adicciones. 29, 256-267. doi:10.20882/adicciones.959.

Golpe, S., Isorna, M., Barreiro, C., Braña, T. y Rial, A. (2017). Consumo intensivo de alcohol en adolescentes: prevalencia, conductas de riesgo y variables asociadas. Adicciones, 29, 268-277. doi:10.20882/adicciones.932.

Goodman, R., Meltzer, H. y Bailey, V. (2003). The Strengths and Difficulties Questionnaire: a pilot study on the validity of the self-report version. International Review of Psychiatry, 15, 173-177. doi:10.1080/0954026021000046137.

Green, M. J., Leyland, A. H., Sweeting, H. y Benzeval, M. (2013). Socioeconomic position and adolescent trajectories in smoking, drinking, and psychiatric distress. The Journal of Adolescent Health, 53, 202-208.e2. doi:10.1016/j.jadohealth.2013.02.023.

Gueorguieva, R., Wu, R., O’Connor, P. G., Weisner, C., Fucito, L. M., Hoffmann, S.,... O’Malley, S. S. (2014). Predictors of abstinence from heavy drinking during treatment in COMBINE and external validation in PREDICT. Alcoholism, Clinical and Experimental Research, 38, 2647-2656. doi:10.1111/acer.12541.

Hibell, B., Guttormsson, U., Ahlström, S., Balakireva, O., Bjarnason, T., Kokkevi, A. y Kraus, L. (2012). The 2011 ESPAD Report: Substance Use Among Students in 36 European Countries. Sweden: The Swedish Council for Information on Alcohol and Other Drugs (CAN). Recuperado de http://www.can.se/contentassets/8d8cb78bbd28493b9030c65c598e3301/the_2011_espad_ report_full.pdf.

Hill, K. G., White, H. R., Chung, I. J., Hawkins, J. D. y Catalano, R. F. (2000). Early adult outcomes of adolescent binge drinking: person- and variable-centered analyses of binge drinking trajectories. Alcoholism, Clinical and Experimental Research, 24, 892-901. doi:10.1111/j.1530-0277.2000.tb02071.x.

Hingson, R. W., Zha, W. y Weitzman, E. R. (2009). Magnitude of and trends in alcohol-related mortality and morbidity among U.S. college students ages 18-24, 19982005. Journal of Studies on Alcohol and Drugs, Supplement $16,12-20$.

Jessor, R. (1991). Risk behavior in adolescence: a psychosocial framework for understanding and action. The Journal of Adolescent Health, 12, 597-605.

Johnston, L. D., O’Malley, P. M., Miech, R. A., Bachman, J. G. y Schulenberg, J. E. (2016). Monitoring the future na- tional survey results on drug use, 1975-2015: Overview, key findings on adolescent drug use. Ann Arbor: Institute for Social Research, The University of Michigan. Recuperado de http:/ / monitoringthefuture.org/pubs/monographs/ mtf-overview2015.pdf.

Johnston, L. D., Bachman, J. G. y Schulenberg, J. E. (2012). Monitoring the future national survey results on drug use, 1975-2011. (Vol. Volume I: Secondary School Students.). Institute for Social Research, University of Michigan; 2012. Recuperado de http://www.monitoringthefuture.org/pubs/monographs/mtf-vol1_2011. pdf.

Kandel, D. B., Johnson, J. G., Bird, H. R., Weissman, M. M., Goodman, S. H., Lahey, B. B.,... Schwab-Stone, M. E. (1999). Psychiatric comorbidity among adolescents with substance use disorders: findings from the MECA Study. Journal of the American Academy of Child and Adolescent Psychiatry, 38, 693-699. doi:10.1097/00004583-19990600000016.

Kim, M. S., Kim, J. J. y Kwon, J. S. (2001). Frontal P300 decrement and executive dysfunction in adolescents with conduct problems. Child Psychiatry and Human Development, 3, 93-106. doi:10.1023/A:1012299822274.

Kuntsche, E., Gabhainn, S.N., Roberts, C., Windlin, B., Vieno, A., Bendtsen, P., ... Wicki, M. (2014). Drinking motives and links to alcohol use in 13 European countries. Journal of Studies on Alcohol and Drugs, 75, 428437. doi:10.15288/jsad.2014.75.428.

Laird, R. D., Jordan, K. Y., Dodge, K. A., Pettit, G. S. y Bates, J. E. (2001). Peer rejection in childhood, involvement with antisocial peers in early adolescence, and the development of externalizing behavior problems. Development and Psychopathology, 13, 337-354.

Lanza, S. T. y Collins, L. M. (2006). A mixture model of discontinuous development in heavy drinking from ages 18 to 30: the role of college enrollment. Journal of Studies on Alcohol, 67, 552-561. doi:10.15288/jsa.2006.67.552.

Lopez Villalba, F. J., Rodriguez Garcia, P. L., Garcia Canto, E. y Perez Soto, J. J. (2016). Relationship between sport and physical activity and alcohol consumption among adolescents students in Murcia (Spain). Archivos Argentinos de Pediatria, 114, 101-106. doi:10.5546/aap.2016. eng.101.

Marmorstein, N. R., Iacono, W. G. y Malone, S. M. (2010). Longitudinal associations between depression and substance dependence from adolescence through early adulthood. Drug and Alcohol Dependence, 107, 154-160. doi:10.1016/j.drugalcdep.2009.10.002.

Maslowsky, J. y Schulenberg, J. E. (2013). Interaction matters: quantifying conduct problem $\mathrm{x}$ depressive symptoms interaction and its association with adolescent alcohol, cigarette, and marijuana use in a national sample. Development and Psychopathology, 25, 1029-1043. doi:10.1017/S0954579413000357. 
McCambridge, J., McAlaney, J. y Rowe, R. (2011). Adult consequences of late adolescent alcohol consumption: a systematic review of cohort studies. PLoS Medicine, 8, e1000413. doi:10.1371/journal.pmed.1000413.

O’Malley, P. M. y Johnston, L. D. (2002). Epidemiology of alcohol and other drug use among American college students. Journal of Studies on Alcohol. Supplement 14, 2339.

Patrick, M. E. y Schulenberg, J. E. (2010). Alcohol use and heavy episodic drinking prevalence and predictors among national samples of American eighth- and tenthgrade students. Journal of Studies on Alcohol and Drugs, 71, 41-45.

Patrick, M. E., Yeomans-Maldonado, G. y Griffin, J. (2016). Daily reports of positive and negative affect and alcohol and marijuana use among college student and nonstudent young adults. Substance Use E $\mathcal{E}$ Misuse, 51, 54-61. do i:10.3109/10826084.2015.1074694.

Paykel, E. S., Myers, J. K., Lindenthal, J. J. y Tanner, J. (1974). Suicidal feelings in the general population: a prevalence study. The British Journal of Psychiatry, 124, 460-469. doi:10.1192/bjp.124.5.460.

Pesola, F., Shelton, K. H., Heron, J., Munafo, M., Hickman, M. y van den Bree, M. B. M. (2015). The developmental relationship between depressive symptoms in adolescence and harmful drinking in emerging adulthood: The role of peers and parents. Journal of Youth and Adolescence, 44, 1752-1766. doi:10.1007/s10964-015-0295-z.

Pesola, F., Shelton, K. H., Heron, J., Munafo, M., Maughan, B., Hickman, M. y van den Bree, M. B. M. (2015). The mediating role of deviant peers on the link between depressed mood and harmful drinking. The Journal of Adolescent Health, 56, 153-159. doi:10.1016/j.jadohealth.2014.10.268.

Pilgrim, C. C., Schulenberg, J. E., O’Malley, P. M., Bachman, J. G. y Johnston, L. D. (2006). Mediators and moderators of parental involvement on substance use: a national study of adolescents. Prevention Science, 7, 75-89. doi:10.1007/s11121-005-0019-9.

Pitkänen, T., Lyyra, A. L. y Pulkkinen, L. (2005). Age of onset of drinking and the use of alcohol in adulthood: a follow-up study from age 8-42 for females and males. Addiction, 100, 652-661. doi: 10.1111/j.13600443.2005.01053.x.

Plan Nacional sobre Drogas. (2016). Encuesta sobre el uso de drogas en enseñanzas secundarias en España (ESTUDES) 2014-2015. Madrid, España: Ministerio de Sanidad, Servicios Sociales e Igualdad. Recuperado de http:/ / www.pnsd.msssi.gob.es/profesionales/sistemasInformacion/sistemaInformacion/pdf/2016_ESTUDES_2014-2015.pdf.

Poulin, C., Hand, D., Boudreau, B. y Santor, D. (2005). Gender differences in the association between substance use and elevated depressive symptoms in a gen- eral adolescent population. Addiction, 100, 525-535. doi:10.1111/j.1360-0443.2005.01033.x.

Richter, M., Kuntsche, E., de Looze, M. y Pförtner, T. K. (2013). Trends in socioeconomic inequalities in adolescent alcohol use in Germany between 1994 and 2006. International Journal of Public Health, 58, 777-784. doi:10.1007/s00038-013-0486-x.

Romero, E., Rodríguez, C., Villar, P. y Gómez-Fraguela, X. A. (2017). Intervención sobre problemas de conducta tempranos como prevención indicada del consumo de drogas: Siete años de seguimiento. Adicciones, 29, 150162. doi:10.20882/adicciones.722.

Rüütel, E., Sisask, M., Värnik, A., Värnik, P., Carli, V., Wasserman, C.,... Wasserman, D. (2014). Alcohol consumption patterns among adolescents are related to family structure and exposure to drunkenness within the family: Results from the SEYLE Project. International Journal of Environmental Research and Public Health, 11, 1270012715. doi:10.3390/ijerph111212700.

Sajber, D., Tahiraj, E., Zenic, N., Peric, M. y Sekulic, D. (2016). Alcohol drinking among Kosovar adolescents: An examination of gender-specific sociodemographic, sport, and familial factors associated with harmful drinking. Substance Use E Misuse, 51, 533-539. doi:10.3109/10 826084.2015.1122064.

Saraceno, L., Heron, J., Munafo, M., Craddock, N. y van den Bree, M. B. M. (2012). The relationship between childhood depressive symptoms and problem alcohol use in early adolescence: findings from a large longitudinal population-based study. Addiction, 107, 567-577. doi:10.1111/j.1360-0443.2011.03662.x.

Schulenberg, J., O’Malley, P. M., Bachman, J. G., Wadsworth, K. N. y Johnston, L. D. (1996). Getting drunk and growing up: trajectories of frequent binge drinking during the transition to young adulthood. Journal of Studies on Alcohol, 57, 289-304.

Sobeck, J., Abbey, A., Agius, E., Clinton, M. y Harrison, K. (2000). Predicting early adolescent substance use: do risk factors differ depending on age of onset? Journal of Substance Abuse, 11, 89-102. doi:10.1016/S08993289(99)00022-X.

Tahiraj, E., Cubela, M., Ostojic, L., Rodek, J., Zenic, N., Sekulic, D. y Lesnik, B. (2016). Prevalence and factors associated with substance use and misuse among Kosovar adolescents; Cross sectional study of scholastic, familial, and sports-related factors of influence. International Journal of Environmental Research and Public Health, 13, E502. doi:10.3390/ijerph13050502.

Terry-McElrath, Y. M., O’Malley, P. M. y Johnston, L. D. (2011). Exercise and substance use among American youth, 1991-2009. American Journal of Preventive Medicine, 40, 530-540. doi:10.1016/j.amepre.2010.12.021.

Tilton-Weaver, L. C., Burk, W. J., Kerr, M. y Stattin, H. (2013). Can parental monitoring and peer manage- 
M. Bousoño, S. Al-Halabí, P. Burón, M. Garrido, E. M. Díaz-Mesa, G. Galván, L. García-Álvarez, Á. Velasco, C. Wasserman, V. Carli, C. Hoven, M. Sarchiapone, D. Wasserman, M. Bousoño, M. P. García-Portilla, C. Iglesias, P. A. Sáiz, J. Bobes.

ment reduce the selection or influence of delinquent peers? Testing the question using a dynamic social network approach. Developmental Psychology, 49, 2057-2070. doi:10.1037/a0031854.

Vanyukov, M. M., Tarter, R. E., Kirisci, L., Kirillova, G. P., Maher, B. S. y Clark, D. B. (2003). Liability to substance use disorders: 1. Common mechanisms and manifestations. Neuroscience and Biobehavioral Reviews, 27, 507-515. doi: 10.1016/j.neubiorev.2003.08.002.

Wallace, J. M., Yamaguchi, R., Bachman, J. G., O’Malley, P. M., Schulenberg, J. E. y Johnston, L. D. (2007). Religiosity and adolescent substance use: The role of individual and contextual influences. Social Problems, 54, 308-327. doi:10.1525/sp.2007.54.2.308.

Wasserman, C., Hoven, C. W., Wasserman, D., Carli, V., Sarchiapone, M., Al-Halabi, S.,... Postuvan, V. (2012). Suicide prevention for youth-a mental health awareness program: lessons learned from the Saving and Empowering Young Lives in Europe (SEYLE) intervention study. BMC Public Health, 12, 776. doi:10.1186/14712458-12-776.

Wasserman, D., Carli, V., Wasserman, C., Apter, A., Balazs, J., Bobes, J.,... Hoven, C. W. (2010). Saving and empowering young lives in Europe (SEYLE): a randomized controlled trial. BMC Public Health, 10, 192. doi:10.1186/1471-2458-10-192.

Wells, J. E., Horwood, L. J. y Fergusson, D. M. (2004). Drinking patterns in mid-adolescence and psychosocial outcomes in late adolescence and early adulthood. Addiction, 99, 1529-1541. doi:10.1111/j.13600443.2004.00918.x.

White, H. R., Johnson, V. y Buyske, S. (2000). Parental modeling and parenting behavior effects on offspring alcohol and cigarette use. A growth curve analysis. Journal of Substance Abuse, 12, 287-310.

White, H. R., Labouvie, E. W. y Papadaratsakis, V. (2005). Changes in substance use during the transition to adulthood: A comparison of college students and their noncollege age peers. Journal of Drug Issues, 35, 281-306. doi:10.1177/002204260503500204.

Wilsnack, R. W., Vogeltanz, N. D., Wilsnack, S. C., Harris, T. R., Ahlstrom, S., Bondy, S.,... Weiss, S. (2000). Gender differences in alcohol consumption and adverse drinking consequences: cross-cultural patterns. Addiction, 95, 251-265.

World Health Organization. (2014). Global status report on alcohol and health 2014. Genova: World Health Organization.

World Health Organization. (2015). Global School-Based Student Health Survey. Recuperado de http://www. who.int/chp/gshs/GSHS_Questionnaire_Core_2009_ Spanish.pdf.

Wray-Lake, L., Maggs, J. L., Johnston, L. D., Bachman, J. G., O’Malley, P. M. y Schulenberg, J. E. (2012). Associ- ations between community attachments and adolescent substance use in nationally representative samples. The Journal of Adolescent Health, 51, 325-331. doi:10.1016/j. jadohealth.2011.12.030.

Yaogo, A., Fombonne, E., Lert, F. y Melchior, M. (2015). Adolescent repeated alcohol intoxication as a predictor of young adulthood alcohol abuse: The role of socioeconomic context. Substance Use E Misuse, 50, 1795-1804. doi:10.3109/10826084.2015.1058824.

Zung, W. W. (1971). A rating instrument for anxiety disorders. Psychosomatics, 12, 371-379. doi:10.1016/S00333182(71)71479-0. 